\title{
MISZELLEN
}

\section{Die Stifterfiguren am Sakramentshaus der Urbanskirche in Unterlimpurg}

\author{
von HeLMUT WÖRNER
}

In der Schausammlung des Hällisch-Fränkischen Museums in Schwäbisch Hall sind zwei Stifterfiguren ausgestellt, die aus der Urbanskirche ${ }^{1}$ in Schwäbisch Hall-Unterlimpurg stammen: die Skulptur einer Frau und die eines Mannes. Bernhard Decker ${ }^{2}$ datiert diese (die Dame) in das Ende des 14. Jahrhunderts beziehungsweise (den Mann) in die Zeit um 1430/1440. Beide Figuren wurden dem Museum 1988 von der Evangelischen Kirchengemeinde als Leihgaben zur Verfügung gestellt. Die Dame war bei der Dachstuhlerneuerung 1982 im Mauerwerk der Chorsüdwand oberhalb der Gewölbekappen gefunden worden. Der Stifter stand bis zur Renovierung der Kirche im Jahr 1980 auf der Konsole links neben der in die Nordwand des Kirchenschiffs eingelassenen Heilig-Grab-Nische $^{3}$. Dieser Tragstein kann schon allein wegen der Haltung des Dargestellten nach links - also abgewendet von der Nische - nicht der ursprüngliche Standort der Statuette gewesen sein.

Ein Archivalienfund im Staatsarchiv Ludwigsburg ${ }^{4}$ zeigt Letztere zusammen mit einer Frauenskulptur, deren Gesichtszüge der 1982 im Gewölbe der Urbanskirche entdeckten Stifterin beziehungsweise der im Wimperg als Relief wiedergegebenen Veronika mit dem Schweißtuch ähneln, als Begleitfiguren des Sakramentshauses der Unterlimpurger Kirche. Die weibliche Figur (heraldisch rechts) ist an der Konsole mit dem gespiegelten Wappen der Herren von Hohenlohe ${ }^{5}$ und

1 Die Urbanskirche ist eigentlich eine Marienkirche.

2 Die Bildwerke des Mittelalters und der Frührenaissance 1200-1565. Bearbeitet von Bernhard Decker. Hg. vom Hällisch-Fränkischen Museum Schwäbisch Hall (Bestandskataloge des Hällisch-Fränkischen Museums Schwäbisch Hall 1). Sigmaringen 1994. S. 34 ff. und S. 46 ff.

3 Vgl. Daniela Naumann: Die Urbanskirche in Schwäbisch Hall. In: WFr 90/91 (2006/07) S. 143 ff.

4 StA Ludwigsburg B 113 I Bü 2198. Der 1593 angelegte Band enthält neben den Zeichnungen der Grabmäler von Mitgliedern des Hauses Limpurg auf der Comburg, in Unterlimpurg, Bamberg, Würzburg, Speckfeld, Obersontheim und Gaildorf auch eine Abbildung des Sakramentshauses der Urbanskirche mit zwei Stifterfiguren. Die Beschriftungen stammen von der Hand des Limpurger Schreibers Christoph Fröschel $(\dagger 1604)$.

5 Aufgrund der Spiegelung des hohenlohischen Wappens sind die beiden Wappenschilde an den Tragsteinen als Allianzwappen zu verstehen. Die Leoparden im hohenlohischen Wappen schreiten 


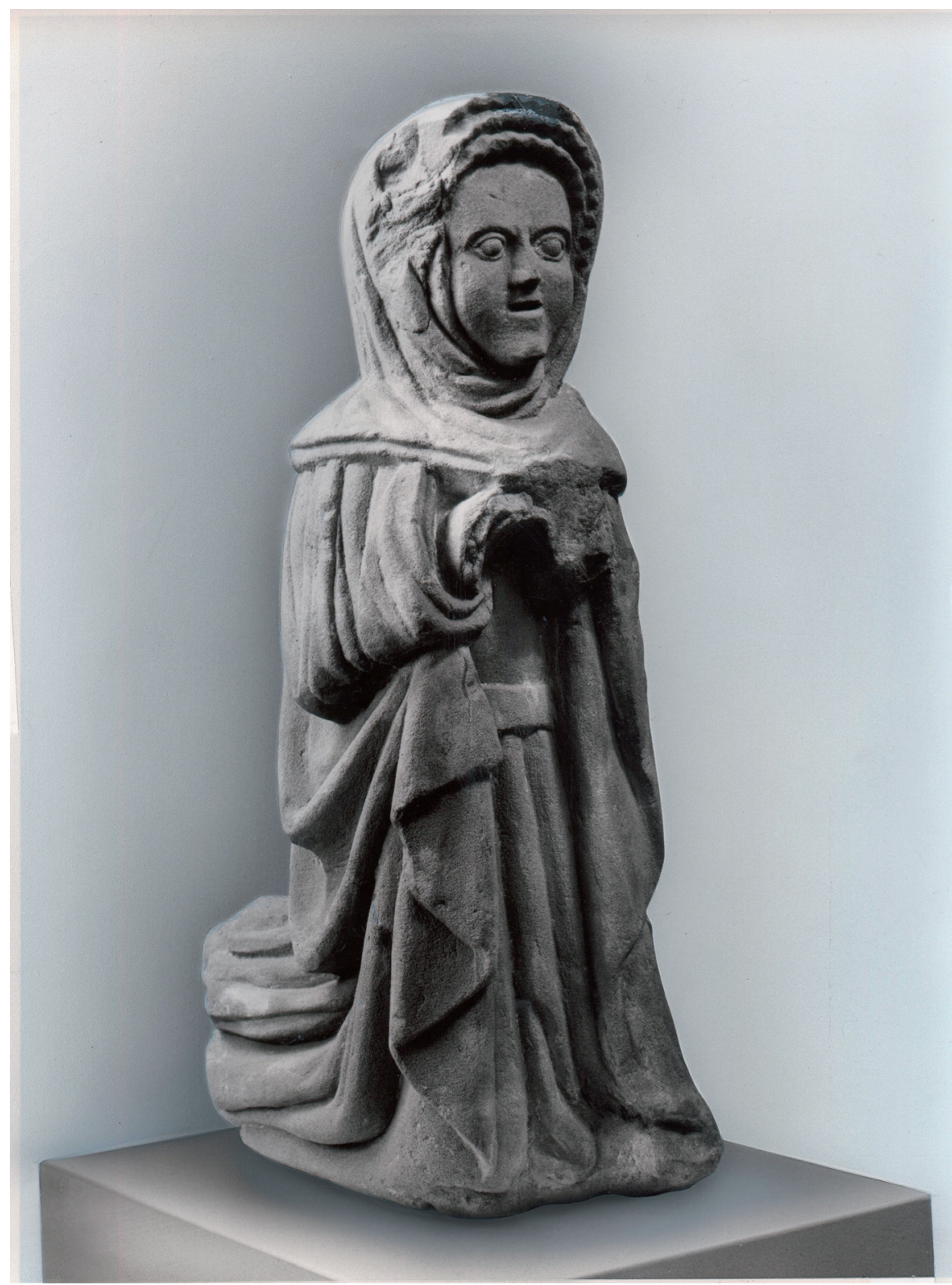

Abb. 1 Adlige Stifterin aus der Urbanskirche in Schwäbisch Hall-Unterlimpurg, Sandstein, Ende 14. Jahrhundert, Inv. Nr. 88/201 (Aufnahme: Margit Kern, Schwäbisch Hall) 


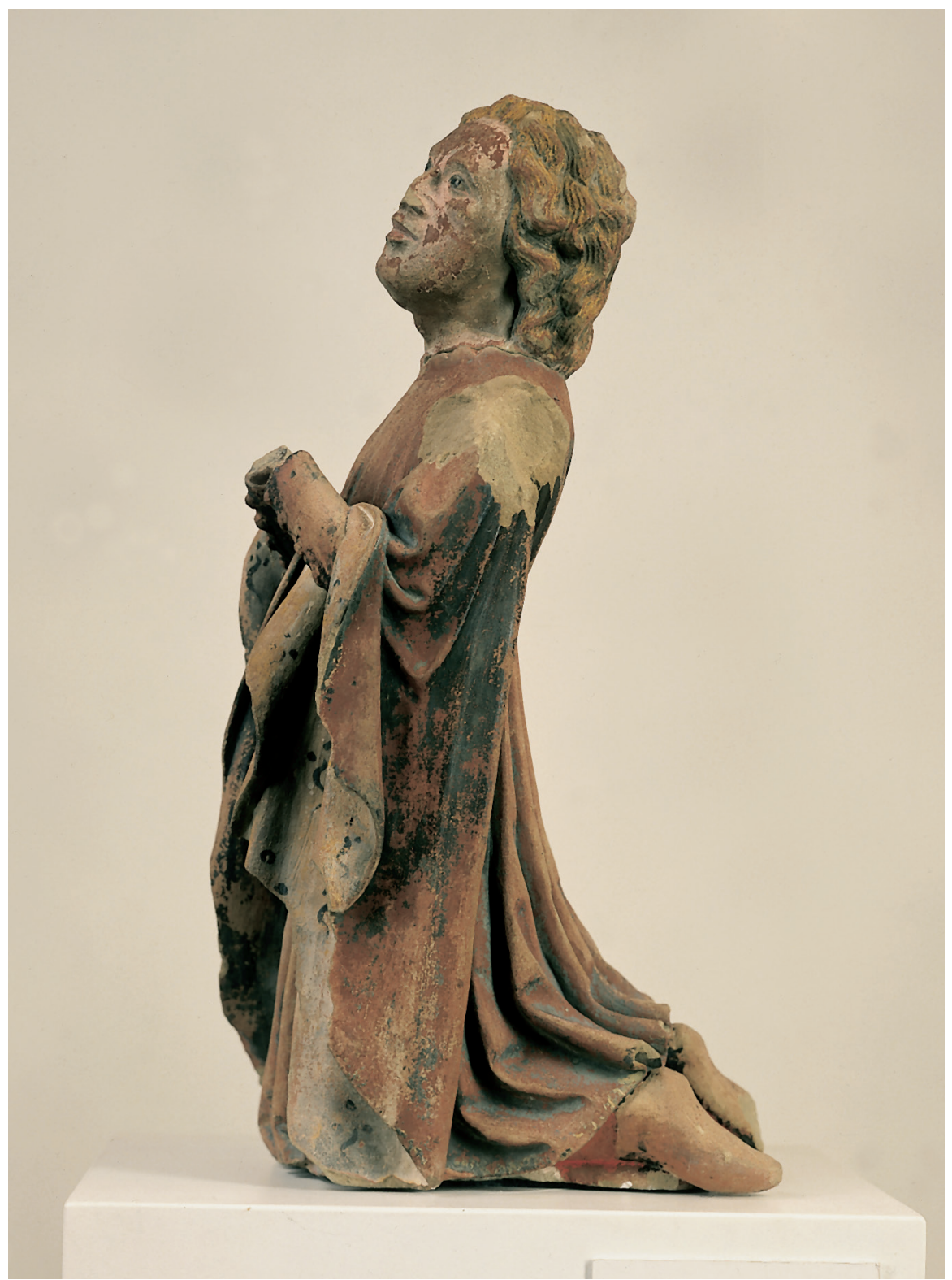

Abb. 2 Adeliger Stifter vom Sakramentshaus der Urbanskirche in Schwäbisch Hall-Unterlimpurg, ein Schenk von Limpurg, Sandstein, Reste einer farbigen Fassung, 1430/1440, Inv. Nr. 88/200 (Aufnahme: Margit Kern, Schwäbisch Hall) 
am unteren Rand des Archivales handschriftlich mit Elisabeth $v$ [on] Hohelohe Stiftung ${ }^{6}$ bezeichnet. Das Wappenschild an der Konsole der Stifterfigur (heraldisch links) zeigt die fünf limpurgischen Streitkolben $(3: 2)$. Noch heute weisen Abbrüche und Ankervertiefungen an den Ecken rechts und links unten auf die ehemals am Sakramentshaus vorhandenen beiden Tragsteine hin.

Der Vergleich der lavierten Tuschezeichnung aus dem Jahr 1593 mit den beiden Statuetten im Hällisch-Fränkischen Museum ergibt Folgendes:

Bei der männlichen Figur gibt es eine Reihe von Übereinstimmungen, auch von Details wie zum Beispiel den Knöpfen oder dem Gürtel des Obergewandes, die nahelegt, dass der Zeichner diese Figur aus eigener Anschauung kannte.

Bei der Stifterin dagegen ist, abgesehen von der Körperhaltung, nur eine spezifische Übereinstimmung festzustellen, nämlich die Kopfbedeckung, der so genannte Kruseler. Einen solchen trägt auch die adlige Dame im Hällisch-Fränkischen Museum, allerdings bis auf den Rüschenrand von einer weiten Kragenkapuze bedeckt.

Die neuesten Forschungen zur Baugeschichte der Urbanskirche ${ }^{7}$ gehen davon aus, dass die weibliche Sandsteinskulptur, die in der südlichen Chorwand eingemauert war, beim Umbau des Kirchenschiffes Mitte des 15. Jahrhunderts dorthin „entsorgt“" wurde. Folglich kann der Zeichner diese, stilistisch dem Meister des figürlichen Schmuckes am Sakramentshaus zuzuordnende Bildhauerarbeit Ende des 16. Jahrhunderts nicht gesehen haben.

\section{Wer waren die Stifterin und der Stifter?}

Aufgrund der abgebildeten Wappen und des handschriftlichen Vermerks auf der Zeichnung stellten die Ende des 16. Jahrhunderts am Sakramentshaus der Urbanskirche stehenden Stifterfiguren Elisabeth von Hohenlohe (um 1375-1445) und einen Schenken von Limpurg dar. Das nach der stilkritischen Bewertung nach 1385 in die nördliche Chorwand eingefügte Sakramentshaus ${ }^{8}$ könnte auf eine Stiftung Elisabeth von Hohenlohes zurückgehen ${ }^{9}$, vielleicht anlässlich ihrer

nicht wie üblich nach heralidsch rechts, sondern nach heraldisch links, sie bewegen sich auf das Limpurger Wappen zu. Das bedeutet, dass die Figuren auf den Konsolen in einer Beziehung zueinander stehen.

6 Vgl. Anm. 4.

7 Vgl. Naumann (wie Anm. 3).

8 Die dendrochronologische Untersuchung der Dachstuhlhölzer bestätigt diese Einordnung. 1385 wurde der Chor der Kirche eingewölbt und erhielt seine heutige Form. Als Fälldatum der Dachstuhlhölzer wurde das Jahr 1385 ermittelt.

9 Vgl. Gerd Wunder, Max Schefold, Herta Beutter: Die Schenken von Limpurg und ihr Land (FWFr 20). Sigmaringen 1982. - Elisabeth von Hohenlohe gebar ihrem Mann elf Kinder. 1413, kurz vor dem Tod Schenk Friedrichs III., brachte sie ihm noch eine reiche Erbschaft ein. Von ihrem 1412 im Krieg gefallenen Bruder Johann erbte sie die halbe Herrschaft Speckfeld (heute Landkreis Kitzingen). Die andere Hälfte der einträglichen Ländereien konnte Elisabeth 1435 von ihren Ver- 


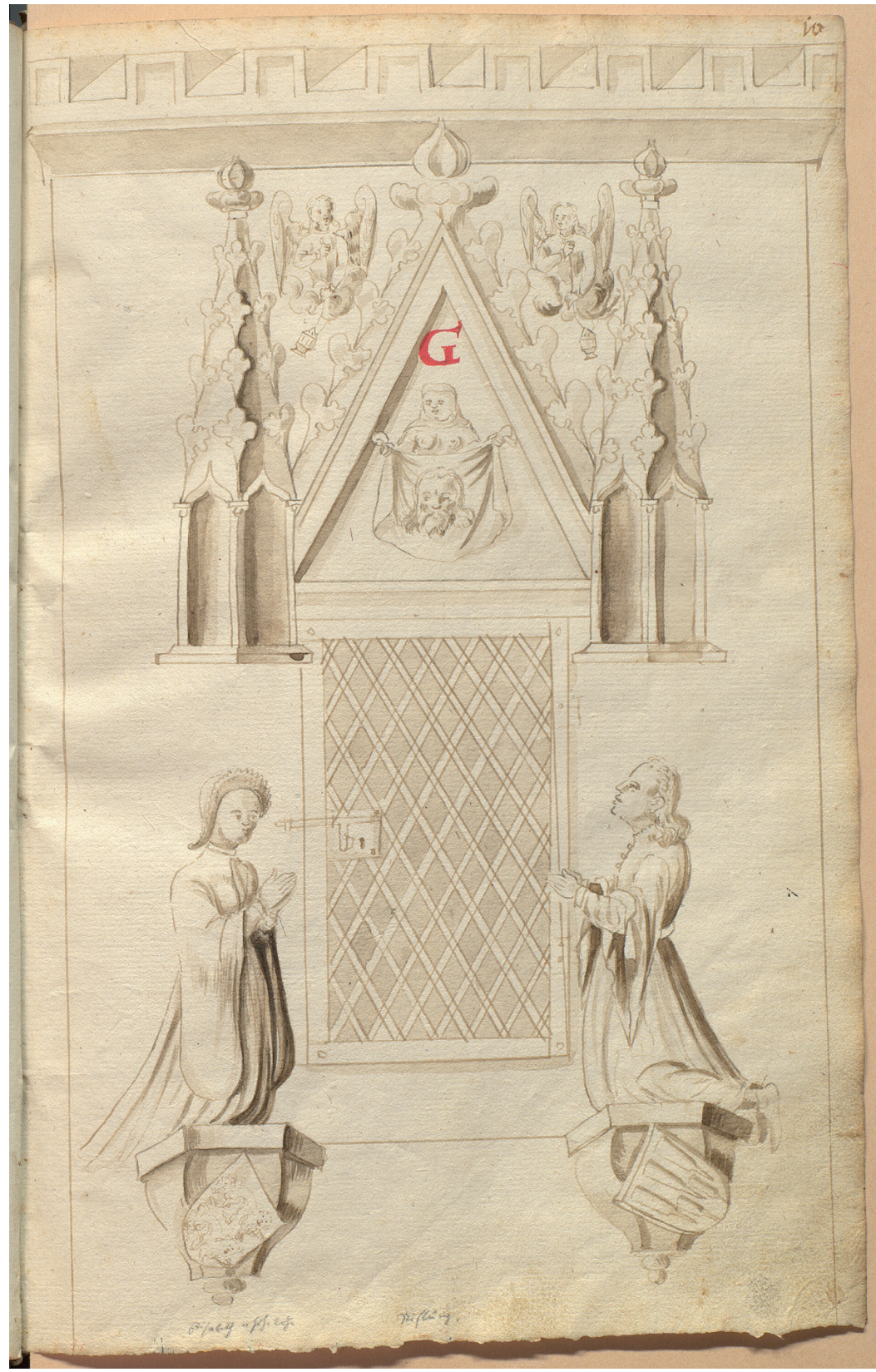

Abb. 3 Sakramentshaus der Unterlimpurger Urbanskirche, lavierte Tuschezeichnung, Ende 16. Jahrhundert (StA Ludwigsburg B 113 I Bü 2198) (Aufnahme: Staatsarchiv Ludwigsburg) 
Vermählung im Jahr 1394 mit Friedrich III. von Limpurg (um 1370-1414) oder eines anderen denkwürdigen Ereignisses in der Schenkenfamilie.

Allerdings fragt sich, ob die sehr starr und etwas altertümlich wirkende weibliche Stifterfigur aus der Urbanskirche, die in der Mittelalterabteilung des Hällisch-Fränkischen Museums ausgestellt ist, wirklich Elisabeth von Hohenlohe darstellt. Daniela Naumann interpretiert die Dame als Utta von Weinsberg ${ }^{10}$. Utta von Weinsberg war die Mutter Schenk Friedrichs III., des Ehemannes von Elisabeth von Hohenlohe.

Um 1430 erfuhr die Urbanskirche mit der nördlichen Erweiterung des Kirchenschiffes eine neue Umbau- und Ausstattungsphase. Damals wurde die Nordwand des Schiffes um ca. drei Meter nach außen versetzt. Seither steht die Westseite des Turmes nicht mehr frei, sondern schließt das nördliche Seitenschiff nach Osten hin ab. Dazu kamen eine Grablegungsnische im neuen Nordschiff und möglicherweise ein Altar an der westlichen Turmwand. Maßgeblich für diese Erneuerung war Elisabeths sehr kunstsinniger Sohn Friedrich V. (1400-1474), der seine geistlichen Ämter aufgegeben hat, um die Regentschaft der Herrschaft Limpurg zu übernehmen. Elisabeth, um die 60 Jahre alt, dürfte damals noch auf der Limpurg gelebt haben; 1435 siedelte sie nach Speckfeld über ${ }^{11}$. Sie hatte die Herrschaft mit großer Umsicht geführt und den Sohn sicherlich auch finanziell beim Ausbau seiner Kirche unterstützt ${ }^{12}$. Vielleicht wollte Friedrich V., der 1437 die vornehme Gräfin Susanna von Thierstein, die Tochter des Pfalzgrafen des Stifts Basel, ehelichte, seiner Mutter und sich mit den Stifterfiguren ein Denkmal setzen, und zwar am vornehmsten Platz der Kirche, der nach katholischem Verständnis dort ist, wo das Allerheiligste (die konsekrierten Hostien) aufbewahrt wird. Doch letztlich muss offenbleiben, ob es sich bei der Figur um ihn oder seinen Vater, Schenk Friedrich III., handelt.

Die um 1430/1440 entstandene Stifterstatuette ist eine Bildhauerarbeit von hoher Qualität. Sie weist Spuren einer farbigen Fassung auf.

Vielleicht war auch die Skulptur der Stifterin bemalt. Die Zeichnung zeigt sie in einen Mantel mit langen weiten Ärmeln gehüllt und den Kopf mit einem Kruseler bedeckt. Der strenge Faltenwurf des Gewandes und der Kopfputz verleihen der Stifterin die ihrem Alter entsprechende Würde ${ }^{13}$.

Unklar ist bis heute, wann die Figuren und die Konsolen am Sakramentshaus entfernt worden sind, und wo die weibliche Stifterfigur abgeblieben ist.

wandten um 11.000 Gulden erwerben. Danach verließ sie die Limpurg und verlegte ihren Lebensmittelpunkt auf die Burg Speckfeld bei Markt Einersheim.

10 Naumann (wie Anm. 3), S. 167.

11 Vgl. Anm. 9.

12 Wie Anm. 9. Elisabeth von Hohenlohe übersiedelte 1435, nach dem Kauf der halben Herrschaft Speckfeld, nach Speckfeld und wohnte dort bis zu ihren Lebensende 1445.

13 Zu Stifterbildern allgemein vgl. Hans Wentzel: Stifterbilder der Zeit um 1400 in Württembnerg. In: WFr NF 20/21 (1939/40) S. 240ff. 